\title{
A proof of the concept of the in-flight launch of unmanned aerial vehicles in a search and rescue scenario
}

\author{
Niels Nauwynck ${ }^{1}$, Haris Balta ${ }^{2}$, Geert De Cubber ${ }^{2}$, Hichem Sahli ${ }^{1,3}$ \\ ${ }^{1}$ Department of Electronics and Informatics, Vrije Universiteit Brussel, Brussels, Belgium \\ 2 Department of Mechanics of the Royal Military Academy, Brussels, Belgium \\ ${ }^{3}$ Interuniversity Microelectronics Centre (IMEC), Heverlee, Belgium
}

\section{ABSTRACT}

This article considers the development of a system to enable the in-flight-launch of one aerial system by another. The article discusses how an optimal release mechanism was developed taking into account the aerodynamics of one specific mothership and child Unmanned Aerial Vehicle (UAV). Furthermore, it discusses the Proportional-Integral-Derivative (PID)-based control concept that was introduced in order to autonomously stabilise the child UAV after being released from the mothership UAV. Finally, the article demonstrates how the concept of a mothership and child UAV combination could be taken advantage of in the context of a search and rescue operation.

\section{Section: RESEARCH PAPER}

Keywords: Unmanned Aerial Vehicles; control; autonomous stabilisation; search and rescue drones; heterogeneous systems

Citation: Niels Nauwynck, Haris Balta, Geert De Cubber, Hichem Sahli, A proof of concept of the in-flight launch of unmanned aerial vehicles in a search and rescue scenario, Acta IMEKO, vol. 8, no. 4, article 4, December 2019, identifier: IMEKO-ACTA-08 (2018)-04-04

Editor: Yvan Baudoin, International CBRNE Institute, Belgium

Received November 23, 2018; In final form February 12, 2019; Published December 2019

Copyright: This is an open-access article distributed under the terms of the Creative Commons Attribution 3.0 License, which permits unrestricted use, distribution, and reproduction in any medium, provided the original author and source are credited.

Funding: This work was supported by the Royal Military Academy of Belgium and the Vrije Universiteit Brussel, Belgium.

Corresponding author: Geert De Cubber, e-mail: geert.de.cubber@rma.ac.be

\section{INTRODUCTION}

\subsection{Problem statement}

As more and more Unmanned Aerial Vehicles (UAVs) are being used in our everyday lives, we are also seeing increasing variety in the systems that are being developed in different applications. This variety should come as no surprise, as it is impossible to create one system that would fit all user needs. Heterogeneous systems (which are all used concurrently) are therefore the way forward. However, this recommendation comes with the problems of interoperability and the search for optimal collaboration strategies between all these different systems.

In this article, we focus on the collaborative action between two UAVs. One acts as a mothership/carrier/launch platform, capable of launching a smaller child system in-flight, which can then be used for close-to-ground missions.

The in-flight launch of one aerial system by another is no easy task and requires the careful consideration of the aerodynamics and control of the two systems. Indeed, in terms of aerodynamics and flight performance, the mothership and child UAVs impose important forces and constraints on one another. These constraints are very different when the aircraft are mechanically interlinked and when they are separated. The autonomous control concept that is implemented for this research experiment on the child UAV needs to be able to cope with these sudden changes in real time at the moment of release in order to prevent a crash.

One example application domain where the use of an in-flight launched rotorcraft could be of great use is the field of search and rescue, where multiple heterogeneous UAVs are being used already [3], [5]. We therefore decided to validate the applicability of the in-flight launch concept in a search and rescue scenario, where a larger carrier UAV is requested to carry a close-in inspection UAV to a certain location (the location at which the incident is supposed to have taken place). The close-in inspection UAV is then released and starts the task of autonomously inspecting a disaster-struck area, searching for human victims by segmenting the visual images it acquires in real time. Based on this analysis, alarms can then be sent to the search and rescue workers when victims are found. In this paper, we do not focus on the autonomous navigation capabilities of the UAV; rather, 
we concentrate on the real-time semantic segmentation methodology.

\subsection{Previous work}

In the field of collaborative UAVs, Lacroix et al. studied in 2007 the multi-agent decision-making process between the different systems in [1]. However, applying these concepts in practical applications and the reality in the field has proven difficult due to the complex nature of operating multiple heterogeneous platforms simultaneously. Serrano et al. proposed in [2] an interoperability concept that enables the messagepassing and collaborative control for multiple heterogeneous UAVs and applied that concept to heterogeneous systems developed within the context of the ICARUS project [3]. They put the interoperability and collaboration concept in [4] into practice in a search and rescue case for the euRathlon challenge [5], where multiple heterogeneous systems (though not all airborne) were validated in a Fukushima-like response simulation scenario. While these operations entailed the use of heterogeneous UAV operations, none of the systems featured an in-flight launch capability.

The in-flight launch of one UAV by a mothership is has been considered most commonly for military operations. Even in 1975, Lempert reported in [13] on a series of flight tests where a target drone was released from a military aircraft. Roberts et al. described in [6] similar flight tests to determine the flight envelope and launch system configuration for which a small (maximum gross weight of $80 \mathrm{lbs}$ ), unpowered UAV glider could be safely launched from the cargo ramp of a C-130 transport aircraft. Safe separation from a C-130 aircraft was demonstrated as well as UAV stability for successful deployment of wings and fly-out. Doi described in [14] the development of an air-launched multi-role UAV system for the Japanese Ministry of Defense. A successful separation between the mothership and child aircrafts was demonstrated here. However, all of the three tests campaigns discussed above considered a manned aircraft as a mothership and only a fixed wing aircraft as the child system. Limited research exists on the particular use of rotorcrafts due to the difficult aerodynamic constraints imposed by the downwash underneath the mothership platform. In May 2018, the US army was the first military agency to present plans to launch UAVs from manned helicopters [15].

In the field of semantic segmentation, important advances have been made in recent years, mainly due to the advent of more powerful machine learning-based approaches. Semantic segmentation assigns a label to each pixel in the output image that represents a specific object from an available class of objects depending on the dataset. With the rise of easy-to-use and lowcost UAVs, a platform can be created for the remote sensing of forests, with an accuracy of $89 \%$ for seven tree types with basic RGB images. This is a real improvement in comparison with previous studies that use expensive hardware [16]. Not only is it possible to calculate the tree type, the tree height and canopy width could be crucial information for search and rescue missions with UAVs [17].

There is a method known as Regions with Convolutional Neural Networks (CNNs), which utilises region-based methods. With results obtained from object detection, it can perform semantic segmentation. Regions with $\mathrm{CNNs}$ is worthwhile because it first extracts a large quantity of object classifications with a selective search and only then computes the CNNs feature. Afterwards, it can classify each region [18].
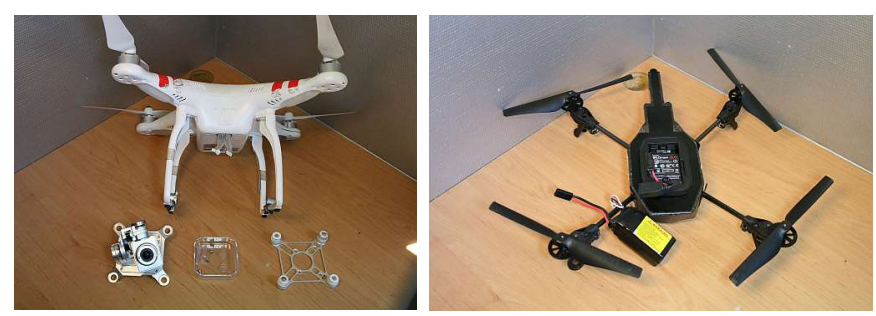

Figure 1. DJI Phantom 2 used as mothership UAV (left); Parrot AR Drone 2.0 used as child UAV (right).

The principle behind Fully Convolutional Networks (FCNs)based semantic segmentation is pixel-to-pixel map learning without the extraction of region proposals. Contrary to CNNs, an FCN only has pooling and convolution layers. Due to these layers, a prediction can be made on inputs of arbitrary sizes. Therefore, the size of the output is dependent on the size of the input instead of a fixed-sized output, which makes it perfect for semantic segmentation or object detection [19].

One semantic segmentation methodology that was specifically adapted to treat visual data coming from UAVs is the SegNet algorithm [20]. The first phase of this algorithm consists of up-sampling the input using the transferred pool to produce the sparse feature map(s). The second phase involves performing convolution to densify the feature map. The last phase is processing the feature maps to pixel-wise classifications with a soft-maximum classifier [21].

The remainder of this paper is organised as follows. Section two discusses the overall conceptual design of the proposed system. Section 3 then focuses on the design of the mechanism that was developed in order to drop the child UAV from the mothership UAV. Section 4 then discusses the control concept that was used to stabilise the child UAV after being dropped. Section 5 then shows how the child UAV can execute a useful mission after being dropped by performing an environmental segmentation in a search and rescue context. Finally, in section 6 , some conclusions and indications for future work are given.

\section{CONCEPTUAL DESIGN}

\subsection{Hardware and software used}

The main aim of this research is to show the concept of the autonomous in-flight launch stabilisation system on commodity hardware multi-copters as opposed to the heavy military systems where in-flight launch systems have already been shown (see the discussion in section 1.2). Therefore, we chose to work with modest, low-cost equipment, as presented here.

The platform used for the parent UAV is a DJI Phantom 2. This ready-to-fly, multi-functional quadcopter is easy to fly, offers precision flight, and has stable hovering - without too much interaction. Throughout this research, this system remained a closed system, where the only communication was done through the included controller. The DJI Phantom 2 is a consumer product not specifically equipped to carry any load but did offer the requirements for the proof of the concept. By removing the pre-installed camera, the total mass of the mothership UAV is $1093 \mathrm{~g}$. The platform used for the child UAV is the Parrot AR Drone 2.0. This UAV is mostly intended for use as a toy, which makes it quite popular and affordable. This UAV has a starting mass of $501 \mathrm{~g}$. By sacrificing security and durability, we were able to reduce the weight by $58 \mathrm{~g}$. However, this weight reduction meant that no protection hull was present during crashes, bringing the lowest mass to $443 \mathrm{~g}$. The Parrot AR Drone 


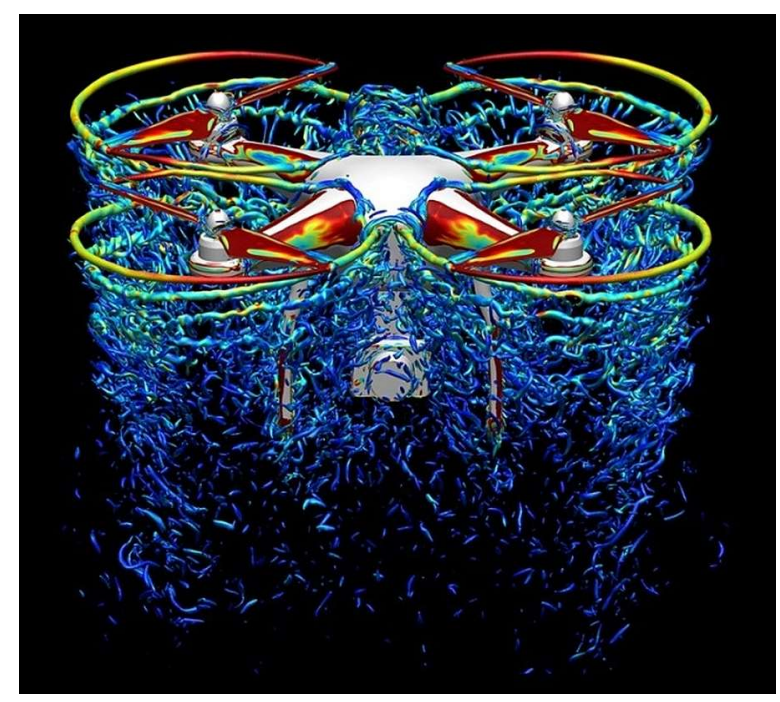

Figure 2. Airflow for around a DJI Phantom quadcopter in hover mode (NASA Ames Graphic/Patricia Ventura Diaz) [23]

2.0 is used frequently in research, because it is programmable in a ROS [7] interface, making use of WiFi communication for input and output. A ROS-driver is provided to create a communication channel with the UAV. This communication driver offers a great deal of functionalities that were used for the in-flight launch software, such as:

a) three-dimensional rotation values from the $\mathrm{X}, \mathrm{Y} \&$ Z axis;

b) magnetometer readings in three-dimensional space;

c) pressure from the barometer;

d) linear velocity in three-dimensional space;

e) linear acceleration in three-dimensional space;

f) estimated altitude;

g) motor pulse width modulation values;

h) forward and downward facing camera stream;

i) yaw, pitch, and roll control.

\subsection{Aerodynamic constraints}

The main problem with the in-flight launch of one rotorcraft from another rotorcraft is the intense turbulent airflow caused by the rotors of the mothership aircraft. One approach to this problem is to experimentally measure the so-called downwash area, as performed in [8]. Another approach is a simulation. High-fidelity computational simulation tools are required to investigate the complex physics of multirotor interactions and interactions with the airframe(s). These simulations require highperformance computing infrastructure and can provide an insight in the turbulence layer underneath the aircraft. Figure 2 gives an example of such an analysis, made by NASA on the Pleiades supercomputer, simulating the airflow underneath the DJI Phantom drone that was used in this study as well [23].

While such simulations are extremely useful, they do not tell the whole story, as they do not incorporate the real-time influence of environmental factors such as wind. Indeed, in order to do so, one would need to measure the wind, calculate the airflow model in real time, and then act upon this updated model. This real time computation capability is currently beyond the capabilities of even modern supercomputers. However, as shown by these research studies [23], these external factors have an important effect on the turbulence layer underneath the aircraft in real-life conditions.
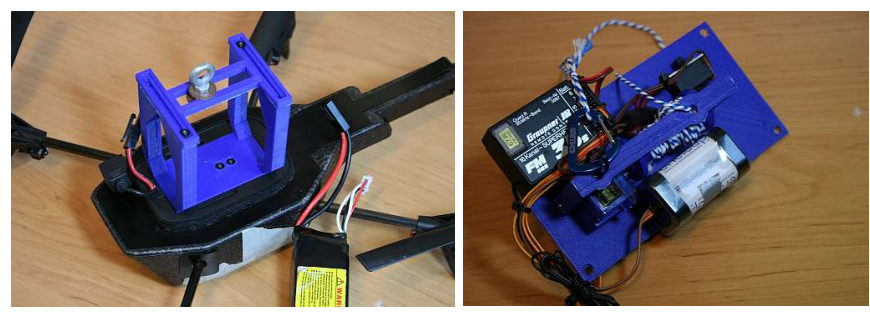

Figure 3. Release mechanism on the child UAV (left), release mechanism on the mothership UAV (right).

Therefore, a modular and flexible design is needed in order for the devices to be able to cope with various environmental conditions. Therefore, we decided to develop a winch-based release mechanism that would allow us to change the release distance between the mothership and child UAVs in terms of environmental parameters.

\section{DESIGN OF THE RELEASE MECHANISM}

As the child UAV still has a task to complete after being launched, as much weight as possible should be left on the mothership UAV. This means that a design was necessary for the actual launch mechanism to hang on the mothership UAV.

A major issue in the process of designing and developing a release mechanism on the child UAV was to prevent any unwanted rotations due to wind, for example, which would cause system instability. Therefore, a child UAV release mechanism was designed, consisting of a base plate and a locking mechanism, terminating in an O-ring to which a hook can be attached. Once the design was fully made, it was $3 \mathrm{D}$ printed. The design turned out to be $44 \mathrm{~g}$. Adding the $44 \mathrm{~g}$ to the $443 \mathrm{~g}$ of the child UAV allowed us to ensure that the child UAV now had a total mass of $487 \mathrm{~g}$. Note that it is technically not possible for a DJI Phantom 2 to support such a payload; therefore, it is necessary for the child UAV to help with lifting its own mass pre-release by spinning its rotors.

Figure 3 shows the results of this design: a lightweight, stern, and rotation resistant component capable of carrying the child UAV.

As discussed above, the child UAV can be carried through an O-ring. This was specifically done to create an easy-to-use launch mechanism on the mothership UAV. The major difficulty on the mothership side was to include a mechanism that can increase or decrease the distance between the mothership and child UAV. As discussed before, due to the effects of turbulence under the mothership aircraft, it is necessary to release the child UAV at a reasonable distance from the mothership UAV, sufficiently far from the turbulence zone.

A winch system consisting of a PCB-controlled servo-motor was developed. Once 3D-printed, the base plate extension creates a functional winch system, as seen in Figure 2. The parent UAV now has the possibility to lower the UAV to any desired launch height from a remote site. The final design of the parent UAV release mechanism has a mass of $245 \mathrm{~g}$, bringing the total mass of the mothership UAV to $1338 \mathrm{~g}$.

\section{AUTONOMOUS STABILISATION}

\subsection{Controller design}

In order to be platform-independent, a new ProportionalIntegral-Derivative (PID) controller was created [22] to take over 


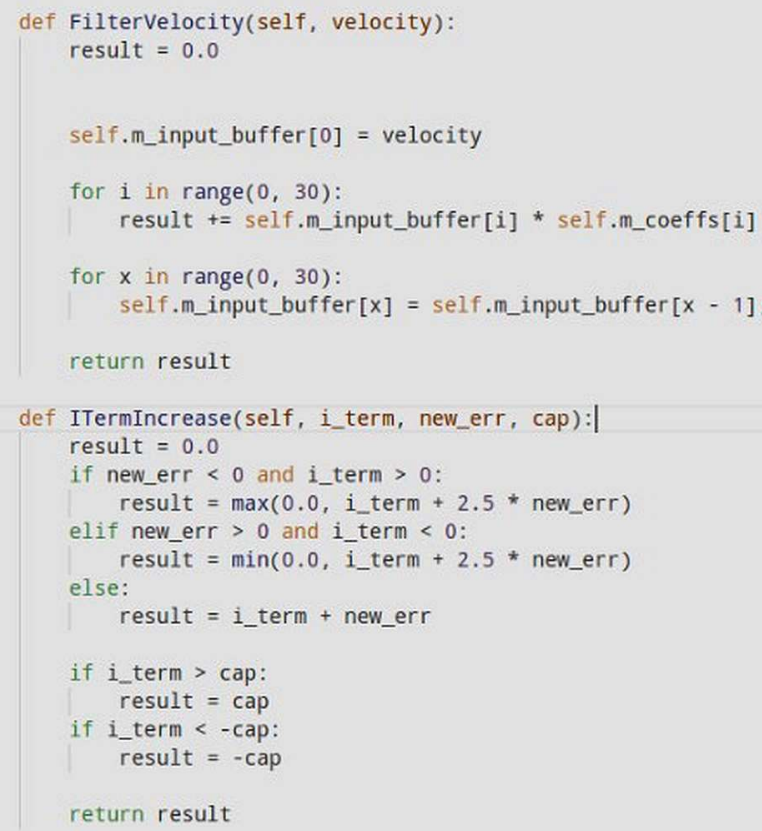

Figure 4. Calculation of the PID controller values.

the default hovering function embedded in the used devices, taking into account the constant turbulence by the mothership UAV. Since we wanted a platform-independent solution, we did not rely on this on the built-in stabilisation method, which also makes use of the downward facing camera. For the creation of the PID controller, a custom package that subscribed to the navigation data and odometry was created. In return, it could publish the necessary yaw, pitch, and roll values, calculated as control commands to stabilise the UAV.

In the implementation, the maximum reference speed of the UAV was limited to 0.6, which prevented it from performing jerky movements. The velocity error was calculated by the difference between the navigation commands of yaw, pitch, and roll and the incoming odometry values. This value was assigned to the proportional gain. The integral gain was calculated based on the previous integral gain and the proportional gain. By using the proportional gain, we were able to determine the integral gain as shown in Figure 4, based on a set limit, the current situation of the error (new err), and the previous integral gain ( $i$ term). Lastly, the derivative gain was calculated by filtering the incoming odometry data.

\subsection{Validation protocol}

In the first phase of the experiment, we wanted to validate the robustness of the implemented control mechanism by manually destabilising the child UAV in-flight.

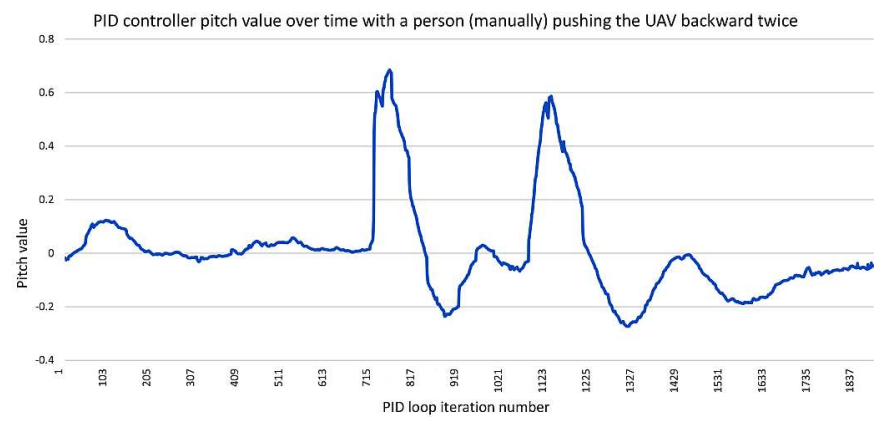

Figure 5. Pitch angle over time, with a person manually pushing the UAV backwards twice.
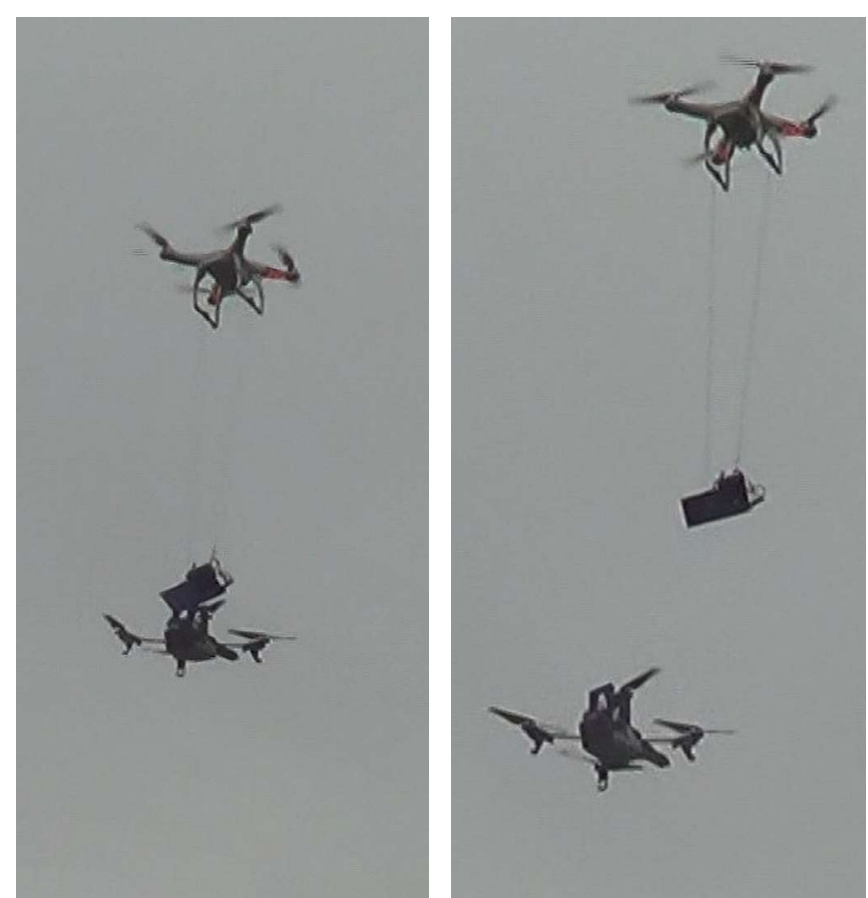

Figure 6. In-flight launch of the child UAV by the mothership UAV: UAVs before launch (left); UAVs after launch (right).

Figure 5 shows the results of such an experiment, where the UAV was pushed backwards twice. The graph shows the value of the pitch angle and clearly shows how the pitch angle is stabilised after each of the disturbances.

Assured by the robustness of the system, actual in-flight launch experiments were performed. In order to study the effect of the turbulence layer, we experimented with difference release altitudes, measured between the mothership and child UAVs. The different separation distances between the both UAVs that were considered were: $140 \mathrm{~cm}, 100 \mathrm{~cm}$, and $60 \mathrm{~cm}$.

\subsection{Behaviour with $140 \mathrm{~cm}$ separation}

Using a $140 \mathrm{~cm}$ launch distance, the PID controller does not need to change the yaw, pitch, or roll values (https://youtu.be/hvxIr1gvgtc). Its only task is increasing the power on all four motors in order to counteract the descent. This is a fairly easy task, and the release therefore goes quite smoothly.

The Pulse-Width Modulation (PWM) motor data displayed in Figure 7 shows the values from the launch till the landing. As seen in the top-left graph, during the lift, the child UAV helps with the overload by throttling the motors to their maximum speed. After the recover loop is initialised, the UAV stabilises.
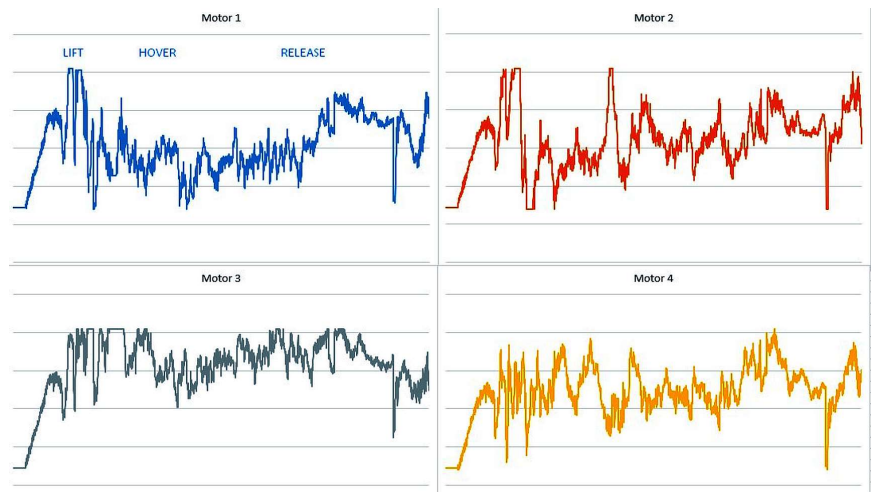

Figure 7. In-flight launch with a $140 \mathrm{~cm}$ distance, with PWM values in the time domain. 

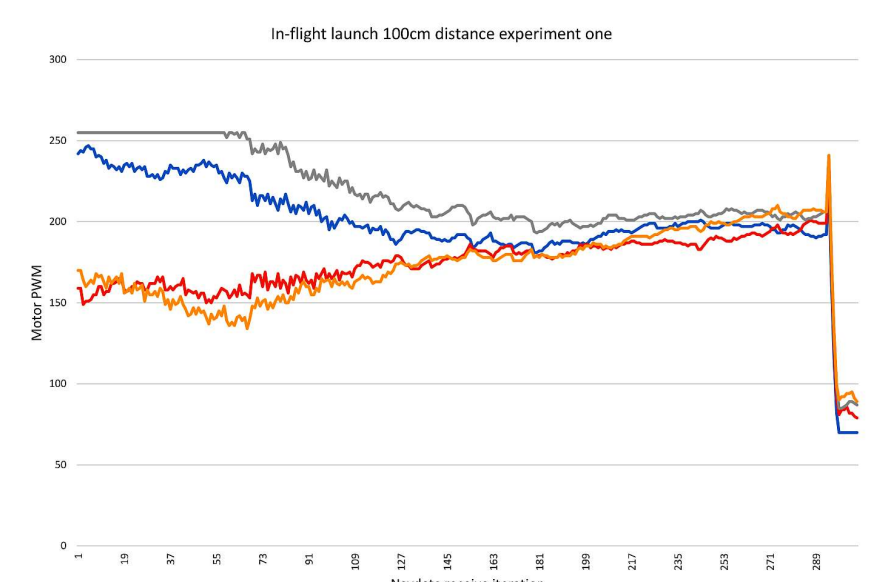

Figure 8. In-flight launch experiment with a $100 \mathrm{~cm}$ distance. The four colours show the PWM values of the four motors.

When releasing the UAV, we can see a clear increase of power on all four motors for counteracting the descent.

\subsection{Behaviour with $100 \mathrm{~cm}$ separation}

Using a $100 \mathrm{~cm}$ launch distance, the behaviour is, in most cases, similar to the previous case, a distance of $140 \mathrm{~cm}$ (https://youtu.be/-HsyfGzBpow). However, we observed that the child UAV sometimes needs to already compensate the prerelease for the extra downward forces induced by the downwash of the mothership UAV. This is the case in the experiment shown in Figure 8. The result is that the PID controller acquires the correct height by lifting its own weight, not relying on the strength of the parent UAV.

\subsection{Behaviour with $60 \mathrm{~cm}$ separation}

Using a $60 \mathrm{~cm}$ launch distance, the distance between the mothership and child UAV becomes very small (https://youtu.be/3Xvp1fMt6tg). As a result, the child UAV is directly within the turbulent area underneath the rotors of the mothership UAV. Consequently, the PID controller is no longer capable of recovering the turbulence induced by the rotors of the mothership UAV, and the child UAV always crashes upon release. Figure 9 displays the PWM values of the motors while helping lift the weight, which was a requirement for in-flight launch. As seen in Figure 9, the motor PWM values are highly variable.

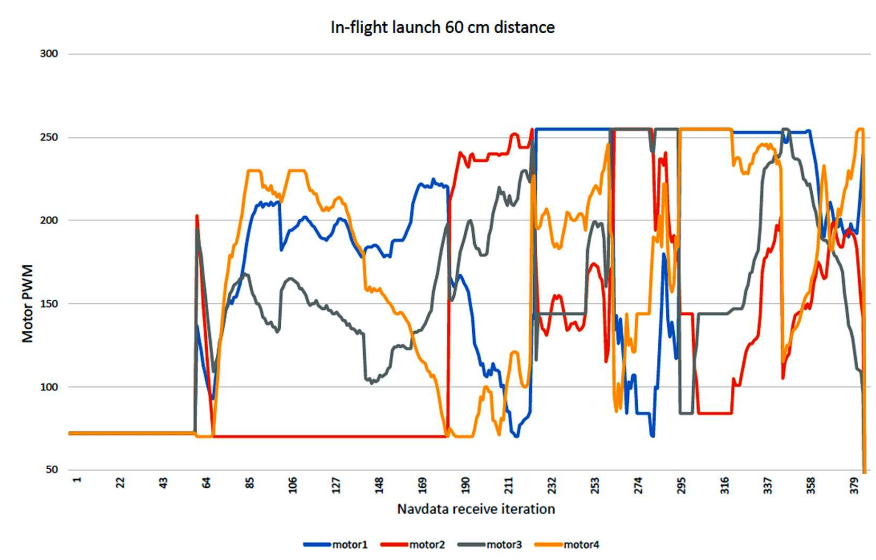

Figure 9. In-flight launch experiment with a $60 \mathrm{~cm}$ distance experiment. The four colours show the PWM values of the four motors.
In all of the four runs, the release was never possible, because the mothership UAV caused turbulence on the child UAV. This turbulence interfered with the spinning propellers of the child UAV which made it move all over the place. Because of the movement of the child UAV, the mothership UAV also started to wiggle, which only increased the movement on the child UAV, repeating this pattern until a crash occurred.

Obviously, this means that we have reached the limits of what was possible with the given platforms and the proposed control and stabilisation paradigm.

\section{VALIDATION IN A SEARCH AND RESCUE SCENARIO}

In order to present a meaningful case for the validation of the proposed system, the field of search and rescue was chosen. This specific domain was not chosen by accident. The specific requirements of search and rescue workers often demand the deployment of multiple heterogeneous robotic tools. Indeed, large fixed wing systems are required to have a permanent eye in the sky and to create a map of the area, while rotorcrafts are generally more suited for outdoor victim searches or dropping rescue kits. Small rotorcrafts are excellent for indoor victim searches. In this context, we envision a search and rescue operation whereby a large UAV launches a smaller one at a specific site such that this small UAV can go and search for victims.

A necessary requirement for using a UAV for victim searches is the capability to detect human survivors in a totally unstructured environment. For scene analysis, using the onboard camera, the UAV has to detect and classify the objects seen by the camera. For this purpose, a Deep Neural Network (DNN) is used to achieve semantic segmentation, assigning a class label to every pixel. A DNN is another form of an artificial neural network that has shown spectacular accuracy on datasets with large feature and solution spaces. Since DNNs often have more vanishing gradient problems and exploding gradient problems, they are harder to train than other networks.

For this application, we use the ENet semantic segmentation algorithm [10], which uses a DNN architecture to provide realtime semantic segmentation for self-driving vehicles. This algorithm runs 18 times faster than existing models by early downsampling, nonlinear operations, changing the decoder size, regularisation, etc. To train from a dataset, a modified version of Caffe was used, which supported all the necessary layers for ENet. This requires a training and testing set whereby the encoder is first trained with pre-labelled objects from the dataset.

After about 75,000 iterations, we noticed a convergence with a minimum training accuracy of $80 \%$. After the decoder had been trained, the encoder was further trained specifically to obtain a training accuracy of $80 \%$. After launching the child UAV from the mothership UAV, the ENET semantic

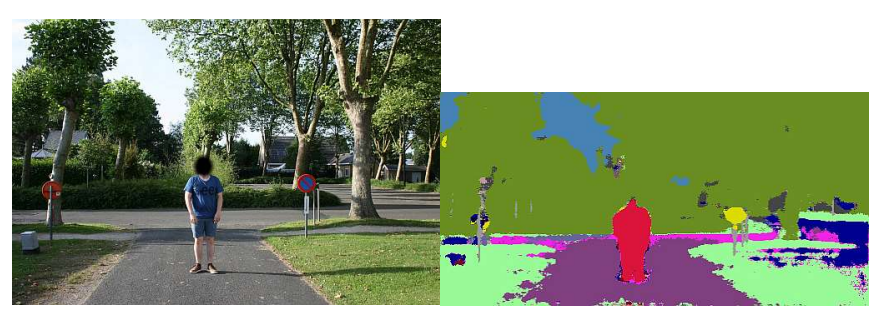

Figure 10. ENet's semantic segmentation input image of a lost person on small road in open countryside. The visual image frame from the child UAV (left), ENet segmentation of the image frame (red=victim) (right). 


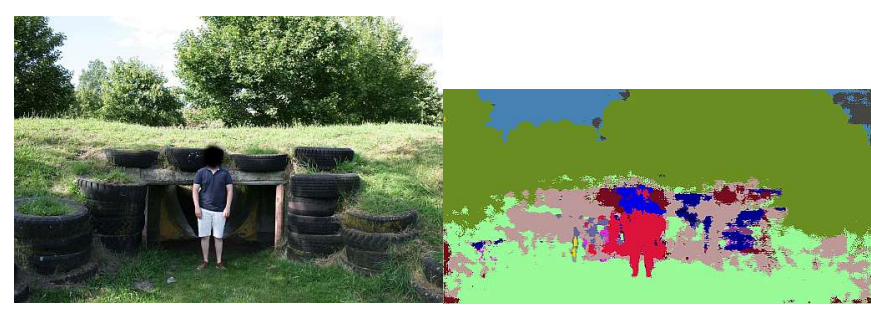

Figure 11. ENet's semantic segmentation output image of a lost person in front of a tunnel. Visual image frame from the child UAV (left), ENet segmentation of the image frame (red=victim).

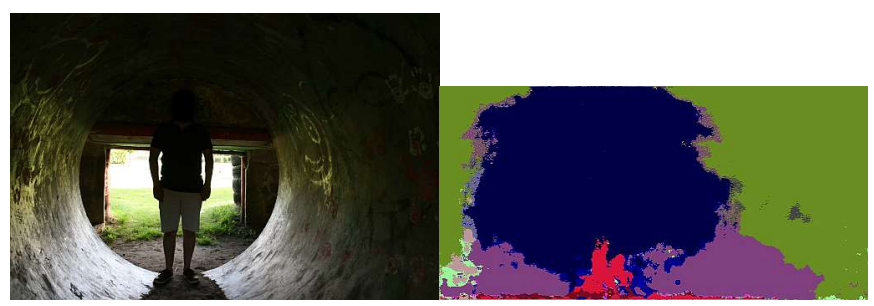

Figure 12. ENet's semantic segmentation output image of a lost person inside a tunnel. Visual image frame from the child UAV (left), ENet segmentation of the image frame (red=victim) (right)

segmentation algorithm was activated on the images of the Parrot AR Drone 2.0 front-facing camera, which has a resolution of 1280 x 720 at $30 \mathrm{fps}$. The first test, shown in Figure 10 shows an example of how the output on a small access road to a building mimics the idea of a small road in open countryside.

The second experiment set can be seen in Figure 11 and 12 and displays the detection possibilities in front of tunnels and shows that while inside a dark tunnel, person detection becomes less obvious.

\section{CONCLUSIONS}

In this article, an in-flight launch concept was proposed for a child rotorcraft UAV by a mothership rotorcraft UAV. The solution was developed not only in theory, but also in practice, by the design of a release mechanism and a control concept in order to stabilise the child UAV after the launch procedure. The system was extensively validated by multiple launch experiments, evaluating the limits of the control concept. Furthermore, a practical use case was elaborated whereby this concept could be put into practice: search and rescue. Therefore, a DNN was implemented in order to perform a semantic segmentation of the video data of the child UAV (after being released in a disaster area by the mothership UAV), enabling autonomous victim search operations.

It must be stressed that the objective of this research work was to provide a proof of concept by using cheap hardware. Future work will thus mainly focus on applying this concept to higher-performing hardware platforms, such that real use cases can be performed.

\section{ACKNOWLEDGEMENT}

This research was institutionally supported by the Royal Military Academy of Belgium and the Vrije Universiteit Brussel, Belgium.

\section{REFERENCES}

[1] S. Lacroix, R. Alami, T. Lemaire, G. Hattenberger, J. Gancet, Decision making in multi-UAVs systems: Architecture and algorithms, in: Multiple Unmanned Aerial Vehicles, ser. Tracts on Advanced Robotics. A. Ollero, I. Maza (editors). Springer, 2007.

[2] D. Serrano López, G. Moreno, J. Cordero, J. Sanchez, Sh. Govindaraj, M. Monteiro Marques, V. Lobo, S. Fioravanti, A. Grati, K. Rudin, M. Tosa, A. Matos, A. Dias, A. Martins, J. Bedkowski, H. Balta, G. De Cubber, Interoperability in a heterogeneous team of search and rescue robots, in: Search and Rescue Robotics-From Theory to Practice. In-Tech, 2017.

[3] G. De Cubber, D. Serrano, K. Berns, K. Chintamani, R. Sabino, R. Ourevitch, D. Doroftei, C. Armbrust, T. Flamma, Y. Baudoin, Search and rescue robots developed by the European ICARUS project, Proc. of the $7^{\text {th }}$ Int. Workshop on Robotics for Risky Environments, 2013.

[4] M. M. Marques, R. Parreira, V. Lobo, A. Martins, A. Matos, Cruz, J. M. Almeida, J. C. Alves, E. Silva, J. Bedkowski, K. Majek, M. Peka, P. Musialik, H. Ferreira, A. Dias, B. Ferreira, G. Amaral, A. Figueiredo, R. Almeida, F. Silva, D. Serrano, G. Moreno, G. D. Cubber, H. Balta, $\mathrm{H}$. Beglerovi, Use of multi-domain robots in search and rescue operations contributions of the ICARUS team to the Eurathlon 2015 challenge, Proc. of OCEANS 2016, April 2016, Shanghai, pp. 1-7.

[5] A. F. T. Winfield, M. P. Franco, B. Bruggemann, A. Castro, M. C. Limon, G. Ferri, F. Ferreira, X. Liu, Y. R. Petillot, J. Roning, F. E. Schneider, E. Stengler, D. Sosa, A. Viguria, Eurathlon 2015: A multi-domain multi-robot grand challenge for search and rescue robots, in: TAROS, ser. Lecture Notes in Computer Science, vol. 9716. Springer, 2016, pp. 351-363.

[6] D. W. Roberts, A. D. Judy, Separation flight tests of a small unmanned air vehicle from a C-130 transport aircraft, Proc. of the NATO STO Meeting, vol. MP-SCI-162-19, 2012. [online] https://www.sto.nato.int/publications/STO $\% 20$ Meeting \%20Proceedings/RTO-MP-SCI-162/MP-SCI-162-19.pdf

[7] M. Quigley, K. Conley, B. P. Gerkey, J. Faust, T. Foote, J. Leibs, R. Wheeler, A. Y. Ng, ROS: An open-source robot operating system, Proc. of the ICRA Workshop on Open Source Software, 2009.

[8] M. Sjholm, N. Angelou, P. Hansen, K. H. Hansen, T. Mikkelsen, S. Haga, J. A. Silgjerd, N. Starsmore, Twodimensional rotorcraft downwash flow field measurements by lidar-based wind scanners with agile beam steering, Journal of Atmospheric and Oceanic Technology 31(4) (2014) pp. 930-937.

[9] D. Doroftei, A. Matos, G. De Cubber, Designing search and rescue robots towards realistic user requirements, in: Advanced Concepts on Mechanical Engineering (ACME), 2014.

[10] A. Paszke, A. Chaurasia, S. Kim, E. Culurciello, ENet: A deep neural network architecture for real-time semantic segmentation, CoRR abs/1606.02147 (2016).

[11] Y. Jia, E. Shelhamer, J. Donahue, S. Karayev, J. Long, R. Girshick, S. Guadarrama, T. Darrell, Caffe: Convolutional Architecture for Fast Feature Embedding, ArXiv e-prints, Jun. 2014. 
[12] M. Cordts, M. Omran, S. Ramos, T. Rehfeld, M. Enzweiler, R. Benenson, U. Franke, S. Roth, B. Schiele, The cityscapes dataset for semantic urban scene understanding, Proc. of the IEEE Conference on Computer Vision and Pattern Recognition (CVPR), 2016.

[13] M. Lempert, Air launch characteristics of the MQM-74C target drone from DC-130 airplane, NTIS Report AD/A004, January 1975.

[14] H. Doi, TACOM - Air-launched multi-role UAV, Proc. of the 24th Congress of International Council of the Aeronautical Sciences, 29 Aug.-3 Sept. 2004, Yokohama, Japan.

[15] J. Judson, US Army to launch drone from helicopter for first time this year, DefenseNews, 1 May 2018.

[16] M. Onishi, T. Ise. Automatic classification of trees using a UAV onboard camera and deep learning. ArXiv e-prints, 1804.10390 (2018).

[17] Y. Seul Lim, H. La, J. Soo Park, M. Hee Lee, M. Wook Pyeon, J.-I. Kim, Calculation of tree height and canopy crown from drone images using segmentation. Journal of the Korean Society of Surveying, Geodesy, Photogrammetry and Cartography 33 (2015) pp. 605-613.
[18] R. Girshick, J. Donahue, T. Darrell, J. Malik, Rich feature hierarchies for accurate object detection and semantic segmentation, Proc. of the IEEE Computer Society Conference on Computer Vision and Pattern Recognition, 2013.

[19] J. Long, E. Shelhamer, T. Darrell, Fully convolutional networks for semantic segmentation, CVPR (2015).

[20] B. Luque, J. R. Morros, J. Ruiz-Hidalgo, Spatio-temporal road detection from aerial imagery using $\mathrm{CNNs}$, Proc. of the International Conference on Computer Vision Theory and Applications, 2017, Porto, Portugal.

[21] V. Badrinarayanan, A. Kendall, R. Cipolla, SegNet: A deep convolutional encoder-decoder architecture for image segmentation. IEEE Transactions on Pattern Analysis and Machine Intelligence 39(12) (2017) pp. 2481-2495.

[22] N. Nauwynck, H. Balta, G. De Cubber, H. Sahli, In-flight launch of unmanned aerial vehicles, Proc. of the International Symposium on Measurement and Control in Robotics ISMCR2018, Sept. 2018, Mons, Belgium.

[23] P. Ventura Diaz, S. Yoon, High-fidelity computational aerodynamics of multi-rotor unmanned aerial vehicles, Proc. of the 2018 AIAA Aerospace Sciences Meeting, AIAA SciTech Forum (AIAA 2018-1266), 2018. 